\title{
SM Otolaryngology Analysis on Correlation between SP and NK-1R and Intranasal Mucosal Contact Point Headache
}

\author{
Guomin Zhao ${ }^{1}$, Jinshu Yin ${ }^{2 *}$, Hong Peng ${ }^{2}$ and Jing Wang ${ }^{3}$ \\ ${ }^{1}$ Department of Otolaryngology Head \& Neck Surgery, Peking University Ninth School of Clinical Medicine, \\ Beijing Shijitan Hospital, Beijng, 100038, China \\ ${ }^{2}$ Department of Otolaryngology Head \& Neck Surgery, Beijing Shijitan Hospital, Capital Medical University, \\ Beijing, 100038, China \\ ${ }^{3}$ Central Laboratory, Beijing Shijitan Hospital, Capital Medical University, Beijng, 100038, China
}

\section{Article Information \\ Received date: Dec 13, 2016 \\ Accepted date: Jan 12, 2017 \\ Published date: Jan 20, 2017 \\ *Corresponding author}

Jinshu Yin, No.10 Tieyi Road, Haidian District, Beijing, 100038, China,

Tel: 0086010 63926665;

Email: yinjinshu55@aliyun.com

Distributed under Creative Commons CC-BY 4.0

Keywords Headache; Contact point; Substance P; NK-1 receptor Founding; Major project of Scientific Research Plan of Beijing Educational Committee (KM201310025026)

Article DOI 10.36876/smotol.1004

\section{OPEN ACCESS}

ISSN: 2574-2418

\section{Abstract}

Objectives: To observe difference of Substance P (SP) and NK-1 Receptor (NK-1R) expression in tissues at contact point and non-contact point among the patients with intranasal mucosal contact point headache and speculate the role of SP and NK-1R in mucosal contact point headache.

Methods: SP and NK-1R in tissues of contact point and non-contact point among 40 patients with intranasal mucosal contact point headache were stained histologically by immunohistochemistry, and the mRNA level was detected by RT-PCR.

Results: SP was located in cytoplasm of acini epithelial cells, distributed in nasal mucosa tissues at both contact point and non-contact point. But stain intensity was significantly increased at contact point $(Z=-2.554$ $\mathrm{P}<0.05)$. NK-1R was located in cytoplasm of acinar epithelial cells, inflammatory cells and nerve fibers of contact point; only in cytoplasm of acinar epithelial cells and nerve fibers of non-contact point. Tinctorial rate of NK-1R increased significantly at contact point $\left(X^{2}=40.438, P<0.01\right)$. mRNA level of SP and NK-1R was up-regulated in nasal mucosa at contact point compared with non-contact point.

Conclusion: Distribution of SP and NK-1R, especially NK-1R, in nasal mucosal tissue at contact point was higher compared with non-contact point. It was suggested that SP and NK-1R were associated with mucosal contact point headache.

\section{Introduction}

Mucosal contact point headache is a newly type of secondary headache in the International Classification of Headache Disorders (ICHD-2) [1], which is usually caused by anatomical abnormalities of the nasal cavity [2]. Clinical resection of mucosal contact point under nasal endoscopy can greatly relieve headache [3], which implies that mechanical compression of mucosal contact point is an important cause of headache. However, few researchers focus on the molecular etiology mechanism of mucosal contact point headache. Substance P (SP) is now widely studied as the pain mediator, which acts by binding to NK-1 receptor (NK-1R) [4]. This paper analyzed the histological location and gene expressions of SP and NK-1R in nasal mucosa of contact point and non-contact point, which suggested that they may play the role in mucosal contact point headache.

\section{Materials and Methods}

The study conformed to Helsinki Declaration and was approved by Ethics Committee in the hospital. Informed consents were obtained from all subjects.

\section{Clinical data}

Forty patients, 24 males and 16 females, were recruited who diagnosed as mucosal contact point headache and scheduled to receive nasal endoscopy from January 2013 to March 2015 at our hospital. The patients, aged 16-65 years with a median age of 31 years, complained of headache in forehead, temporal region, occipital region or periorbital region from 4 months to 10 years with the median of 3 years. The manifestations such as stuffiness, swelling pain, prickling-like pain or compression would be aggravated by catching a cold, poor rest or mental stress. The patients of preoperation were performed conventional nasal endoscopy and high-resolution coronal paranasal sinus CT scan, and surveyed by Eysenck Personality Questionnaire Short Scale China (EPQ-RSC) using the same instructions and explanations [5]. According to the scores of each item, the patients had stable emotions and no personality disorders.

Diagnostic criteria for mucosal contact point headache [1]: (i) intermittent pain located in the periorbital or medial canthal, temporozygomatic regions; (ii) The severity of the headache is 
mediated by gravitational changes. Compared with using placebo or other control, headache disappeared within 5 min following topical application of topical anesthesia to the contact point area; (iii) confirmed presence of mucosal contact point by both nasal endoscopy and sinus CT scan, without acute or chronic nasosinusitis, nasal polyp and nasal cavity nasal tumor; (iv) headache disappeared within $7 \mathrm{~d}$ after resection of mucosal contact point. The inclusion criteria and exclusion criteria were the same as those in previous literatures [6].

\section{Pain intensity assessment}

The criteria were the same as those in previous literatures [3] 0 point was for no headaches, 100 point for unbearable headaches. According to their own headache feeling, patients have given a score. Pain intensity is divided into 5 levels, level 0 ( 0 point) : no headaches; Level 1 ( $1 \sim 20$ points) : have a headache, feel headache when the note; Level $2(21 \sim 40$ points) : have a headache, sometimes ignore headaches; Level 3 ( $41 \sim 60$ points) : have a headache, cannot ignore but can do daily work and life; Level 4 (61 $~ 80$ points) : have a headache, it's hard to concentrate, can only do simple work; Level 5 (81 100 points) : have a headache, can't do anything. Preoperative and postoperative scores difference is used to evaluate the operation effect. Preoperative pain intensity of this group of cases: Level $5-7$ cases, Level 4 - 7 cases, Level 3 - 20 cases, Level 2 - 6 cases, Level 1 - 0 cases.

\section{Surgical methods and sampling sites}

Functional nasal endoscopic surgery was performed under general anesthesia. The surgical approach was chosen based on site of lesion, severity and CT scan for resection of mucosal contact point with preservation of normal anatomic structure of nasal cavity. The patients with nasal septum deviation were operated by submucous correction of nasal septum or septoplasty; spurs or ridges of nasal septum were resected such as those contacted to lateral wall of the nasal cavity; middle turbinplasty was selected for anatomical variations of the middle turbinate; superior turbinate or ethmoidal sinus was resected for olfactory cleft area contact. The uncinate process resection was performed if hypertrophy or pneumatization, and inferior turbinate out fracture or with hypothermia plasma radiofrequency ablation if hypertrophy.

Sampling sites: turbinate mucosa of the contact point as experimental group was collected, while the anterior portion of the middle turbinate mucosa on the affected side was collected for the control group. A patch measuring about $4 \mathrm{~mm} \times 6 \mathrm{~mm}$ was collected from each patient and divided into two parts for immunohistochemical detection and quantitative PCR, respectively. The fresh specimens were immediately placed in liquid nitrogen and preserved at $-80^{\circ} \mathrm{C}$.

\section{Immunohistochemical detection}

Nasal mucosa specimens of the contact point and non-contact point were embedded in paraffin, sectioned, dewaxed, hydrated and subjected to antigen retrieval. The specimens were cultured with mouse anti-SP secondary antibody (ab14184, 1:50, Abcam) or mouse anti-NK-1R primary antibody (ab25445, 1:00, Abcam) at $4^{\circ} \mathrm{C}$ overnight. The following day, HRP-conjugated secondary antibody (DaKo) was applied for $2 \mathrm{~h}$ at room temperature. After rinsed, DAB coloration agent (DaKo) was added for $10 \mathrm{~min}$ to visualize the secondary antibodies when bound to the primary antibody. The specimens then were counterstained by hematoxylin and dehydrated, transparentized, and covered with cover slips. For negative control, PBS buffer was added instead of primary antibody.

Criteria for positive results: Cells stained brown were considered positive. 10 high-power fields of vision for each slice were randomly selected and the percentage of positive cells per 200 cells in each field of vision was calculated. Blind counting was performed and the average was taken for the positive rate. The scoring criteria were 0 point for negative result, 1 point for $1 \%-25 \%$ positive rate, 2 point for $26-50 \%, 3$ point for $51 \%-75 \%$ and 4 point for $76 \%-100 \%$.

\section{Real-time quantitative reverse transcription-PCR}

Reagents: TRIzol, Transcriptor First Strand cDNA Synthesis Kit and Brilliant II SYBR Green QPCR Master Mix (USA), agarose (Biowest, Spain).

Procedures: Total RNA was extracted from the contact point and non-contact point of nasal mucosa by Trizol one-step method. Concentration and purity of the extracted RNA were detected by UV spectrophotometer (Thermo Scientific NanoDrop 2000). First strand cDNA for PCR was generated from $1 \mu \mathrm{g}$ of total RNA using the Transcriptor First Strand cDNA Synthesis Kit. The primers for amplification by real-time fluorescence quantitative PCR were designed by using Primer-BLAST from NCBI and synthesized by Beijing SBS Genetech Co., Ltd. The primers were as follows: SP (157bp) sense ACGACAGCGACCAGATCAAG, antisense AGAGCCTTTAACAGGGCCAC; NK-1R (295bp) sense TGACCGCTACCACGAGCAAGTCTC, antisense ATAGTCGCCGGCGCTGATGAAG; internal reference $\beta$-actin (238bp) sense GGGTCAGAAGGATTCCTATG, antisense GGTCTCAAACATGATCTGGG. Real-time RT-PCR was performed on Applied Biosystems 7900HT real time system using the Brilliant II SYBR Green QPCR Master Mix (USA). The PCR reaction consisted of $12.5 \mu$ l of Brilliant II SYBR Green QPCR Master Mix, $400 \mathrm{nM}$ of forward and reverse primers, $0.376 \mu \mathrm{l}$ diluted reference dye, and $2 \mu \mathrm{l}$ template cDNA. In the end, the volume of reaction system can up to $25 \mu \mathrm{l}$ by pouring the $\mathrm{PCR}$ - grade $\mathrm{H}_{2} \mathrm{O}$ without nuclease. Cycling was performed in the conditions: $10 \mathrm{~min}$ at $95^{\circ} \mathrm{C}$, followed by 40 rounds of $30 \mathrm{~s}$ at $95^{\circ} \mathrm{C}$ and $60 \mathrm{~s}$ at $60^{\circ} \mathrm{C}$. To verify that the used primer pair produced only a single product, a dissociation protocol was added after thermocycling, determining dissociation of the PCR products from $60^{\circ} \mathrm{C}$ to $95^{\circ} \mathrm{C}$. RQ value represented upregulation or downregulation of mRNA expression of SP and NK-1R in contact point and non-contact point; RQ $>1$ indicated upregulation of mRNA expression, while $\mathrm{RQ}<1$ indicated downregulation.

\section{Postoperative outcome}

It is cured when postoperative headache disappeared completely; It is improved when intensity and duration of headache reduced; It is invalid when symptoms did not change significantly.

\section{Statistical analysis}

Statistical analysis was performed using SPSS16.0 software. Discrepancy of preoperative and postoperative headache was compared by paired-samples $\mathrm{T}$ test. The degree of staining for SP expression in the contact point and non-contact point was compared by rank sum test. In addition, the positive rate of staining for NK-1R expression in the contact point and non-contact point was analyzed by chi-square test. A P-value of $<0.05$ was considered statistically significant. 
Table 1: Preoperative and postoperative headache intensity score.

\begin{tabular}{|c|c|c|c|c|c|c|c|c|c|c|c|}
\hline No. & Pre & Post & No. & Pre & Post & No. & Pre & Post & No. & Pre & Post \\
\hline 1 & 90 & 45 & 11 & 85 & 30 & 21 & 90 & 0 & 31 & 75 & 0 \\
\hline 2 & 65 & 0 & 12 & 70 & 65 & 22 & 85 & 45 & 32 & 100 & 0 \\
\hline 3 & 50 & 50 & 13 & 55 & 0 & 23 & 65 & 0 & 33 & 90 & 60 \\
\hline 4 & 90 & 0 & 14 & 60 & 35 & 24 & 35 & 35 & 34 & 45 & 45 \\
\hline 5 & 75 & 40 & 15 & 30 & 30 & 25 & 60 & 0 & 35 & 55 & 20 \\
\hline 6 & 65 & 0 & 16 & 55 & 25 & 26 & 50 & 0 & 36 & 50 & 15 \\
\hline 7 & 75 & 0 & 17 & 50 & 50 & 27 & 35 & 0 & 37 & 30 & 30 \\
\hline 8 & 30 & 0 & 18 & 60 & 0 & 28 & 35 & 35 & 38 & 55 & 0 \\
\hline 9 & 55 & 20 & 19 & 45 & 45 & 29 & 50 & 45 & 39 & 45 & 45 \\
\hline 10 & 40 & 15 & 20 & 50 & 0 & 30 & 50 & 25 & 40 & 60 & 0 \\
\hline
\end{tabular}

\section{Results}

4.1 The nasal endoscopic surgery were followed up for $6 \sim 18$ months. Post-operative: Level 50 cases, Level 41 cases, Level 39 cases, Level 29 cases, Level 14 cases, Level 017 cases. Cure of 17 cases, cure rate is $42.5 \%$; improvement in 12 cases, improvement rate is $30 \%$, the total effective rate was $72.5 \%$. Invalid in 11 cases, no efficiency was $27.5 \% .5$ cases have a headache better in $12 \sim 18$ months after surgery and reply to preoperative levels. The recurrence rate was $12.5 \%$. Preoperative and postoperative patients with headache given scores.

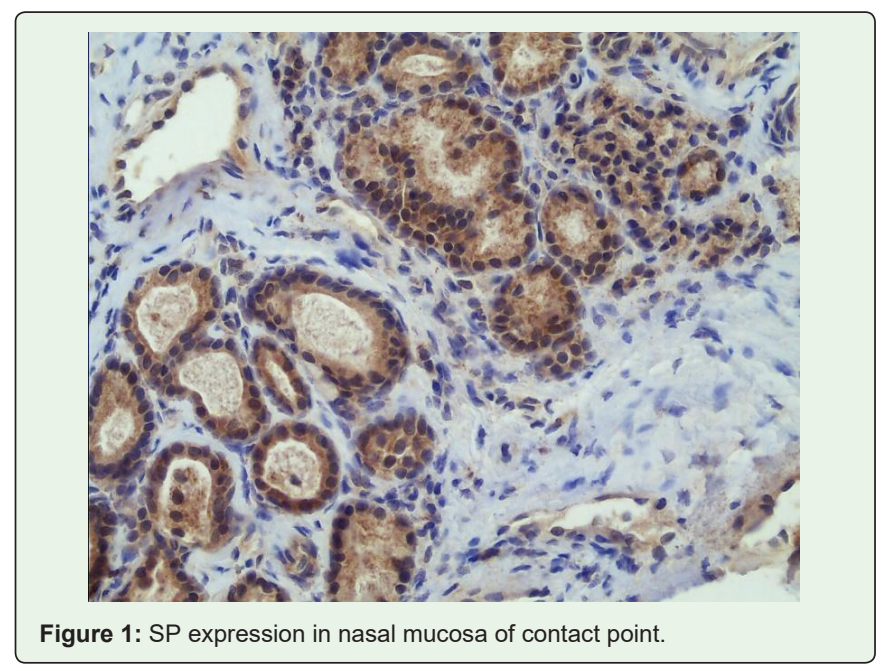

Table 2: Comparisons of degree of staining for SP expression in contact point and non-contact point.

\begin{tabular}{|c|c|c|}
\hline Score & Contact point & Non-contact point \\
\hline 0 & 3 & 3 \\
\hline 1 & 0 & 13 \\
\hline 2 & 8 & 3 \\
\hline 3 & 8 & 13 \\
\hline 4 & 21 & 40 \\
\hline Total & 40 & \\
\hline$Z$ value & & -2.554 \\
\hline P value & & 0.011 \\
\hline
\end{tabular}

Table 3: Comparisons of staining for SP expression in contact point and noncontact point.

\begin{tabular}{|c|c|c|}
\hline & Stained & Not stained \\
\hline Contact point & 38 & 2 \\
\hline Non-contact point & 9 & 31 \\
\hline$X^{2}$ value & & 40.438 \\
\hline P value & & 0.000 \\
\hline
\end{tabular}

(Table 1). Preoperative and postoperative headache was statistically significant difference $(t=7.944, \mathrm{P}<0.01)$. It illustrated that the nasal mucous membrane contact can cause chronic headache.

\section{Histological location of SP and NK-1R in nasal mucosa of contact point and non-contact point}

(1) 37 of 40 specimens of contact point and non-contact point were positive in staining for SP expression, which localized in the cytoplasm of acinar epithelial cells (Figure 1, Figure 2). The degree of staining of SP in contact point and non-contact point was statistically significant difference $(\mathrm{Z}=-2.554, \mathrm{P}<0.05)$ (Table 2$)$ compared by rank sum test.

(2) 38 of 40 specimens of contact point were positive in staining for NK-1R expression, which localized in glandular epithelial cells, inflammatory cells and nerve fibers (Figure 3). 9 of 40 specimens of non-contact point were positive in NK-1R expressions and localized in cytoplasm of acinar epithelial cells and nerve fibers only (Figure 4). Positive rate of NK-1R in contact point and non-contact point was statistically significant difference $\left(\chi^{2}=40.438, \mathrm{P}=0.000\right)$ (Table 3 ) compared by chi-square test.

\section{mRNA expressions of SP and NK-1R in nasal mucosa of contact point and non-contact point}

As shown by real-time fluorescence quantitative PCR, the dissolution curves of SP and NK-1R were unimodal (Figure 5, Figure 6), which indicated no non-specific amplified products. 25 of 40 nasal mucosa specimens of contact point showed upregulation of SP and NK-1R, accounting for $62.5 \%$.

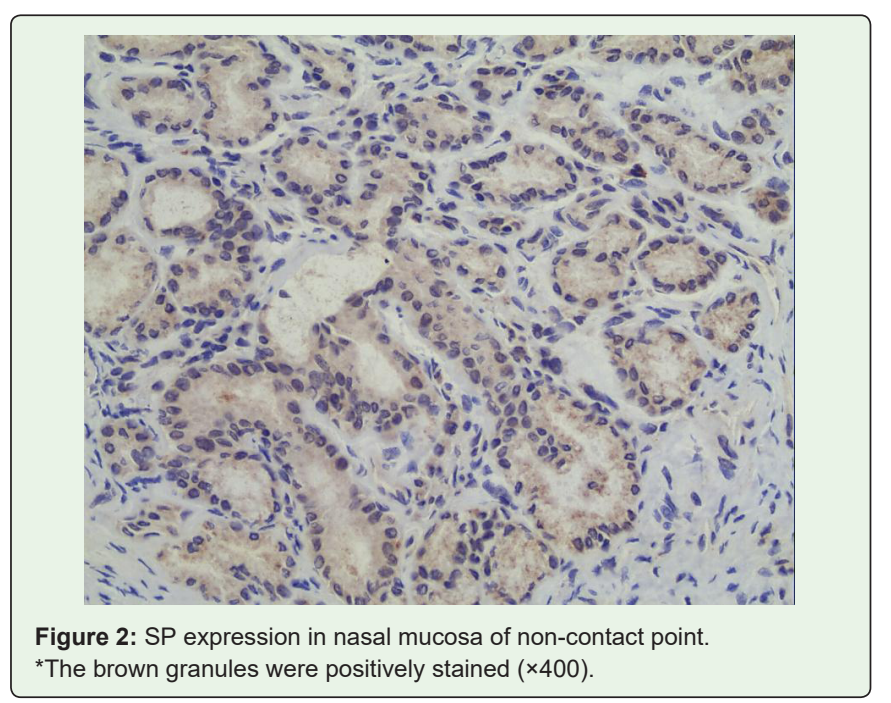

Citation: Zhao G, Yin J, Peng H and Wang J. Analysis on Correlation between SP and NK-1R and Intranasal Mucosal Contact Point Headache. SM 


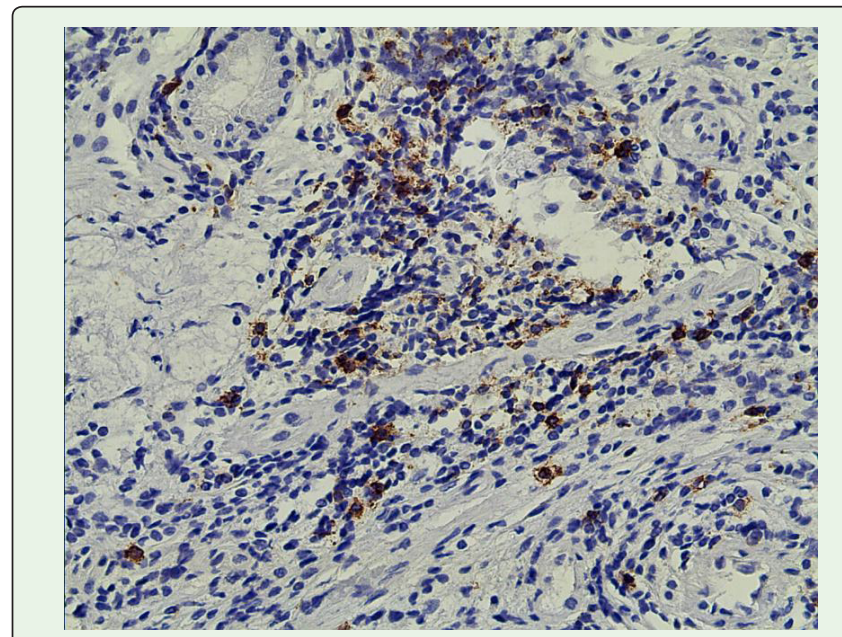

Figure 3: NK-1R expression in nasal mucosa of contact point.

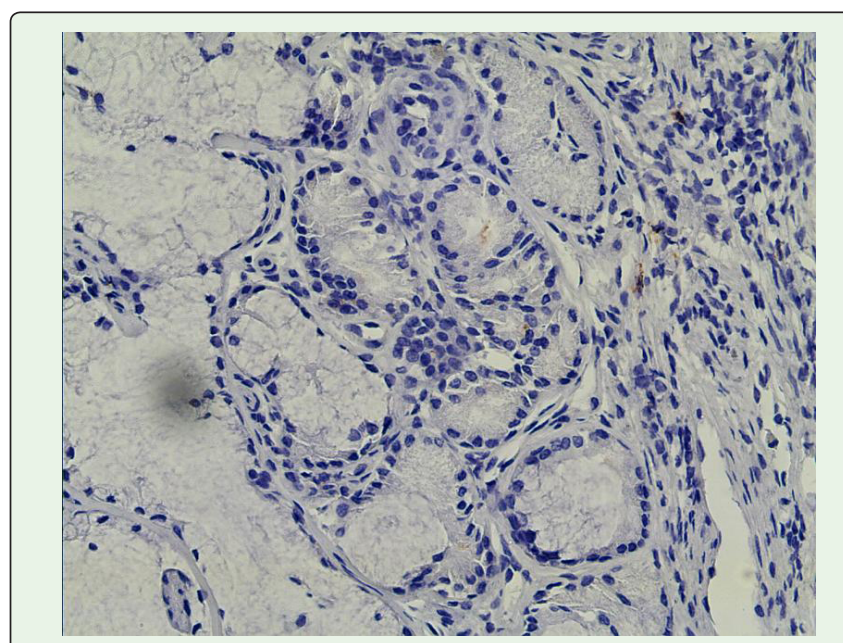

Figure 4: NK-1R expression in nasal mucosa of non-contact point. *The brown granules were positively stained $(\times 400)$.

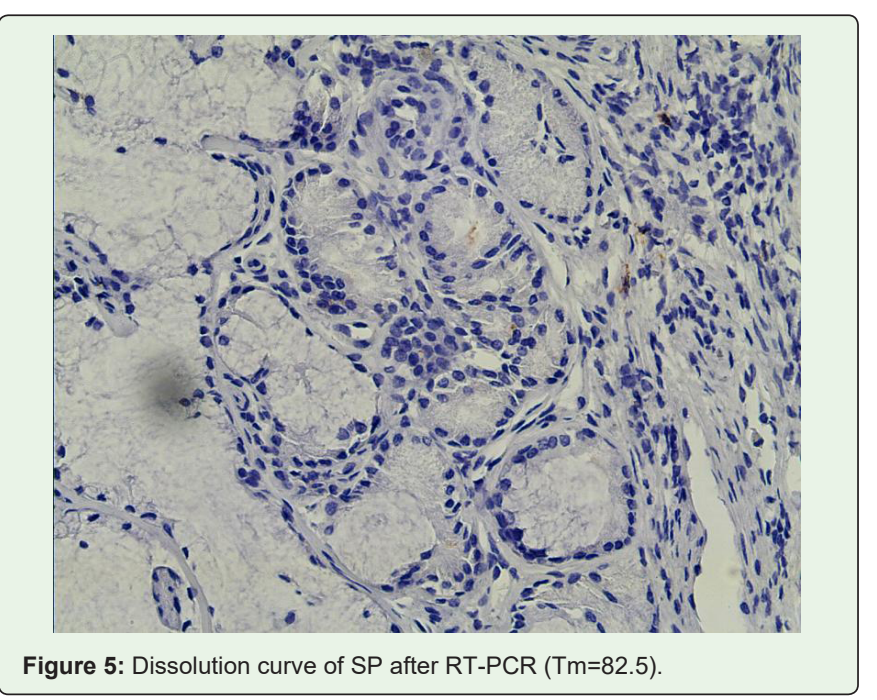

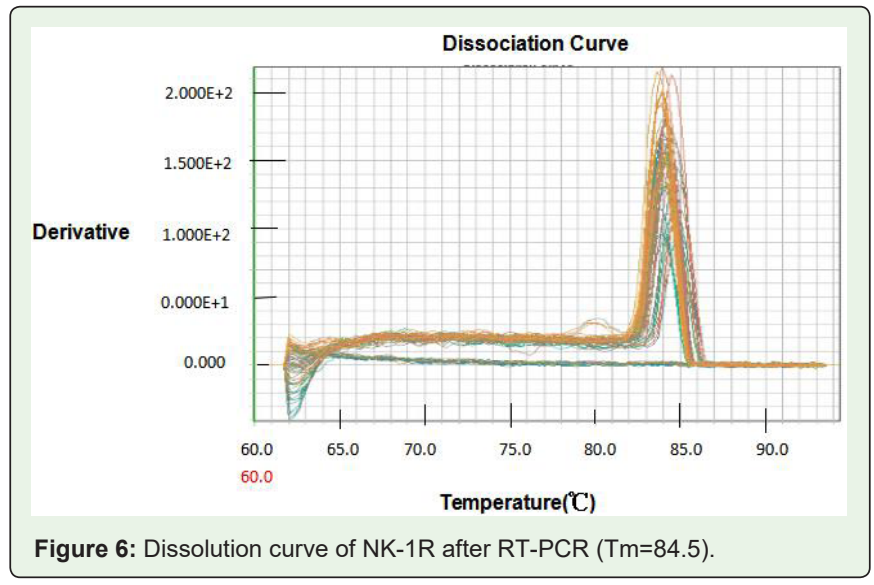

\section{Discussion}

$\mathrm{SP}$ is an undecapeptide encoded by preprotachykinin-A (TAC1) gene [4], which belongs to the tachykinin family of peptides. SP, located in sensory-C fibers in human nasal mucosa, is a neurotransmitter in nocioceptive fibers in the central nervous system [7]. The biological actions of tachykinins are mediated by three receptors, designated Neurokinin (NK)-1, NK-2 and NK-3 [8,9]. The biological action of SP is mainly mediated by the NK-1R [10]. NK-1R, belonging to $\mathrm{G}$ protein-coupled receptor, is encoded by Tachykinin Receptor 1 (TACR1) gene [11]. After binding to the NK-1R, SP contributes to pain transmission [4].

Sensation of pain in several parts of the human body is related to SP and NK-1R. Brown DC et al. [12] found that intrathecal administration of SP-Saponin (a neurotoxin) in dogs with bone cancer selectively destroyed NK-1R expressed in spinal dorsal horn, thereby greatly relieving spontaneous bone cancer pain. After intra-articular injection of NK-1 receptor antagonists into rats with induced arthritis, it was found that the paw withdrawal threshold (pain response) was elevated compared with that before the injection. This indicated that hyperalgesia caused by arthritis was alleviated by preventing the binding of SP to NK-1R [13]. Zheng XF et al. [14] discovered that $\mathrm{NK}-1 \mathrm{R}$ antagonist could reduce the pain caused by osteoporosis due to estrogen deficiency. Zhang $\mathrm{H}$ et al. [15] built the pain model of rats with chronic prostatitis. They detected the tail-flick threshold after injection of complete Freund's adjuvant and found upregulation of SP and NK-1R at posterior horn of L5-S1 spinal cord. It was inferred that the binding of SP to NK-1R caused the activation of astrocytes and enhanced the secretion of proinflammatory cytokines, thereby producing persistent pain.

The pathogenesis of mucosal contact point headache is complex, and several hypotheses are proposed: (1) middle turbinate hypertrophy or pneumatization leads to contact with nasal septum closely, which generates the loss of gap between the two structures, resulting in mechanical compression of the anterior and posterior ethmoidal nerves and hence causing reflex headache [6]; (2) mechanical stress on the nasal mucosal contact point can trigger the release of SP through sensory-C fibers in human nasal mucosa and lead to headache [16]. Baraniuk JN et al. [7] found that SP exists in normal nasal mucosa tissues. Cao YQ et al. [17] pointed out that the generation of moderate to severe pain was closely associated with SP. Antje Welge-Luessen et al. [18] believed that stimulation of the 
receptors in nasal mucosa would cause SP liberation both centrally and peripherally. SP will reach the nasal cavity mucosa via the unmyelinated C-type nerve fibers, where it binds to its receptors to cause pain.

In our study, 37 nasal mucosa specimens of both contact point and non-contact point were positive for SP expression among a total of 40 specimens individually. SP was localized in the cytoplasm of acinar epithelial cells. However, the degree of staining was obviously higher in the specimens of contact point. 38 specimens of 40 contact point were positively stained for NK-1R expression, which was localized in the cytoplasm of glandular epithelial cells and inflammatory cells and nerve fibers. While 9 specimens of 40 noncontact point were positively stained for NK-1R expression, which was localized in the cytoplasm of acinar epithelial cells and nerve fibers. The positive rate of NK-1R expression differed significantly in specimens of contact point and non-contact point. As shown by detection of mRNA expression, 25 out of 40 specimens of contact point had upregulated mRNA expression of SP and NK-1R, and the difference was statistically significant between contact point and noncontact point. NK-1R at the contact point provides the material basis, and its binding to SP triggers the headache.

Cases in this group, incidence of headache was reduced significantly when mucosal contact point was removed by nasal endoscopic surgical. It suggested that mucosal contact point can cause chronic headache. Effectiveness of post-operation was good in short-term (within 12 months). However, during 12-18 months, there were 5 patients with headache returned to preoperative levels. Among them, 2 patients rechecked paranasal sinus CT in our hospital. There was no presence of mucosal contact point in sinus CT scan and ventilation is good, but patients still had headaches prompt. It prompted that contact point oppressed was not the only cause of headaches. High expression of SP and NK-1R in contact point provides the explanation of the causes of headache. Welge Luessen [18] made a 10-year longitudinal study of endonasal surgery for contact point headaches: the effective rate was $85 \%$ after 2 years, and 10 years later fell to $65 \%$ efficient. So long-term follow-up is very important, not only to short-term postoperative headache symptom improvement as a standard for evaluation of curative effect.

Our study showed that the distribution of SP and NK-1R was differential in contact point and non-contact point and suggested that the binding of two was correlated with mucosal contact point headache. Further quantification of SP and NK-1R and the analysis of their regulatory mechanism will provide solid foundation for finding out the methods of clinical diagnosis and even therapy of mucosal contact point headache.

\section{Acknowledgement}

Thank my mentor Yin Jinshu for giving me guidance in the course of my research, thank Dr. Wang Jia for providing clinical data and previous research results, thank Dr. Peng Hong for surgeries support and Wang Jing who works in central laboratory for guidance, thanks all members of our team, there would be no such research without you, thank you!

\section{References}

1. Igarashi $\mathrm{H}$. The International Classification of Headache Disorders: 2nd edition. Cephalalgia. 2004; 24: 9-160.

2. Peng $\mathrm{H}$, Yin J, Ji W. Uninflammatory rhinogenic headache. Zhonghua Er Bi Yan Hou Tou Jing Wai Ke Za Zhi. 2006; 13: 495-497.

3. Wang J, Yin J. Treatment of mucosal contact point headache by nassal endoscopy. Zhonghua Er Bi Yan Hou Tou Jing Wai Ke Za Zhi. 2011; 18: 307-310.

4. Muñoz $M$, Coveñas $R$. Involvement of substance $P$ and the NK-1 receptor in human pathology. Amino Acids. 2014; 46: 1727-1750

5. Mingyi Q, Guocheng W, Rongchun Z, Shen Z. Development of the revised Eysenck personality questionnaire short scale for Chinese (EPQ-RSC) . Xin Li Xue Bao. 2000; 32: 317-323.

6. Wang J, Yin JS, Li JD, Yang L, Duan YL. Clinical observation on relation between nasal mucosa contact point and headache. Zhonghua $\mathrm{Er} \mathrm{Bi}$ Yan Hou Tou Jing Wai Ke Za Zhi. 2012; 47: 317-319.

7. Abu-Bakra M, Jones NS. Does stimulation of nasal mucosa cause referred pain to the face? Clin Otolaryngol Allied Sci. 2001; 26: 430-432.

8. Ebner $\mathrm{K}$, Singewald $\mathrm{N}$. The role of substance $\mathrm{P}$ in stress and anxiety responses. Amino Acids. 2006; 31: 251-272.

9. Ebner K, Sartori SB, Singewald N. Tachykinin receptors as therapeutic targets in stress-related disorders. Curr Pharm Des. 2009; 15: 1647-1674.

10. Muñoz M, Coveñas R. Involvement of substance $P$ and the NK-1 receptor in cancer progression. Peptides. 2013; 48: 1-9.

11. Sharp SI, McQuillin A, Marks M, Hunt SP, Stanford SC, Lydall GJ, et al. Genetic association of the tachykinin receptor 1 TACR1 gene in bipolar disorder, attention deficit hyperactivity disorder, and the alcohol dependence syndrome. Am J Med Genet B Neuropsychiatr Genet. 2014; 165: 373-380.

12. Brown DC, Agnello K. Intrathecal substance P-saporin in the dog: efficacy in bone cancer pain. Anesthesiology. 2013; 119: 1178-1185.

13. Uematsu $T$, Sakai $A$, Ito $H$, Suzuki $H$. Intra-articular administration of tachykinin $\mathrm{NK}_{1}$ receptor antagonists reduces hyperalgesia and cartilage destruction in the inflammatory joint in rats with adjuvant-induced arthritis. Eur J Pharmacol. 2011; 668: 163-168.

14. Zheng XF, Li B, Zhang YH, Yang YH, Meng XY, Jiang SD, et al. Blockade of substance $P$ receptor attenuates osteoporotic pain, but not bone loss, in ovariectomized mice. Menopause. 2013; 20: 1074-1083.

15. Zhang H, Liu LM, Lu GS, Xiong EQ, Li WB, Zhou ZS, et al. Correlation between activation of L5-S2 spinal cord astrocytes and effect of substance $P$ in chronic prostatitis pain. Zhonghua Nan Ke Xue. 2009; 15: 1021-1027.

16. Blumenthal HJ. Headaches and sinus disease. Headache. 2001; 41: 883888

17. Cao YQ, Mantyh PW, Carlson EJ, Gillespie AM, Epstein CJ, Basbaum Al. Primary afferent tachykinins are required to experience moderate to intense pain. Nature. 1998; 392: 390-394

18. Welge-Luessen A, Hauser R, Schmid N, Kappos L, Probst R. Endonasal Surgery for Contact Point Headaches: A 10-Year Longitudinal Study. Laryngoscope. 2003; 113: 2151-2156. 